\title{
Symbol Message Passing Decoding of Nonbinary Spatially-Coupled Low-Density Parity-Check Codes
}

\author{
Emna Ben Yacoub \\ Institute for Communications Engineering \\ Technical University of Munich \\ Munich, Germany \\ emna.ben-yacoub@tum.de
}

\author{
Francisco Lázaro \\ Institute of Communications and Navigation \\ DLR (German Aerospace Center) \\ Wessling, Germany \\ Francisco.LazaroBlasco@dlr.de
}

\author{
Alexandre Graell i Amat \\ Department of Electrical Engineering \\ Chalmers University of Technology \\ Gothenburg, Sweden \\ alexandre.graell@chalmers.se
}

\author{
Gianluigi Liva \\ Institute of Communications and Navigation \\ DLR (German Aerospace Center) \\ Wessling, Germany \\ Gianluigi.Liva@dlr.de
}

\begin{abstract}
The performance of a decoding algorithm, called symbol message passing (SMP) is analyzed, for nonbinary spatially coupled low-density parity-check (LDPC) codes. The SMP algorithm can be seen as a generalization of Gallager $B$ and the binary message passing algorithm by Lechner $e t$ al. to $q$-ary LDPC codes. The analysis is performed via density evolution over the $q$-ary symmetric channel, with $q$ being the field order used for the code construction.
\end{abstract}

\section{INTRODUCTION}

The quest for low-complexity decoding algorithms for lowdensity parity-check (LDPC) codes has recently received a renewed interest, largely triggered by the need of efficient error correction schemes for very high throughput applications. Low-complexity message passing algorithms for binary LDPC codes date back to the seminal work by Gallager [1], who introduced algorithms now known as Gallager A and Gallager B. In [2], an algorithm that allows erasures in the decoder, named Algorithm E, was proposed, with significantly better performance than Gallager B. Another class of decoding algorithms, referred to as binary message passing (BMP), was introduced in [3]. As Gallager's original algorithms, BMP is characterized by the exchange of binary messages between variable nodes (VNs) and check nodes (CNs). However, it exploits channel soft information at the VNs, leading to a much improved performance. An extension of BMP to ternary message alphabets with erasures was studied in [4]. A finitealphabet iterative decoder for the binary symmetric channel was presented in [5]. Low-complexity decoding algorithms have also been considered for nonbinary LDPC codes for both the Gaussian [6]-[8] and the $q$-ary symmetric channel ( $q$-SC) [9]-[14]. In [14], a list message passing decoding algorithm for nonbinary LDPC codes over the $q$-SC was proposed, with performance significantly improving with the list size up to a given size. However, the decoder data flow also increases with the list size. For list size 1, the exchanged messages take values on a $(q+1)$-ary alphabet that includes an erasure message. In
[8], a lookup table-based decoding algorithm that is practical for small values of $q$ was presented. In [15], a novel decoding algorithm for $q$-ary LDPC codes, dubbed symbol message passing (SMP), was introduced, with better performance and similar complexity than the algorithm in [14] for list size 1 .

Spatial coupling has received a great deal of attention in the last years, due to the remarkable threshold saturation effect, i.e., the belief propagation threshold saturates to the maximum a posteriori threshold of the underlying uncoupled ensemble [16], [17]. Threshold saturation for binary LDPC codes under belief propagation (BP) decoding was proved in [18] for the binary erasure channel (BEC) and later in [19] for binary-input memoryless symmetric channels. Threshold saturation for nonbinary spatially coupled low-density paritycheck (SC-LDPC) codes over the BEC was proved in [20]. In [21], one and two bit message passing algorithms for BP decoding of SC-LDPC codes with higher-order modulation were investigated.

In this paper, we extend the analysis of the SMP algorithm of [15] to SC-LDPC codes. We derive the density evolution for protograph-based SC-LDPC codes for transmission over the $q$-SC. The obtained decoding thresholds significantly improve those of the uncoupled ensembles.

\section{Preliminaries}

\section{A. Q-ary symmetric channel}

Consider a $q$-SC with error probability $\epsilon$, input alphabet $\mathcal{X}$ and output alphabet $\mathcal{Y}$, with $\mathcal{X}=\mathcal{Y}=\left\{0, \alpha^{0}, \ldots, \alpha^{q-2}\right\}$, where $\alpha$ is a primitive element of $\mathbb{F}_{q}$. Denote by $X \in \mathcal{X}$ and $Y \in \mathcal{Y}$ the random variables (RVs) associated to the channel input and channel output, respectively, and by $x$ and $y$ their realizations. Then, the transition probabilities of the $q$-SC are

$$
P_{Y \mid X}(y \mid x)= \begin{cases}1-\epsilon & \text { if } y=x \\ \epsilon /(q-1) & \text { otherwise }\end{cases}
$$


The capacity of the $q-\mathrm{SC}$, in symbols per channel use, is

$$
C=1+\epsilon \log _{q} \frac{\epsilon}{q-1}+(1-\epsilon) \log _{q}(1-\epsilon) .
$$

For a given channel output $y$, we introduce the normalized $\log$-likelihood vector, also referred to as $\boldsymbol{L}$-vector,

$$
\boldsymbol{L}(y)=\left[L_{0}(y), L_{1}(y), \ldots, L_{\alpha^{q-2}}(y)\right]
$$

whose elements are obtained as

$$
L_{u}(y)=\log \left(P_{Y \mid X}(y \mid u)\right)-\log (\epsilon /(q-1)) .
$$

From (1), we have

$$
L_{u}(y)= \begin{cases}\mathrm{D}(\epsilon) & \text { if } u=y \\ 0 & \text { otherwise }\end{cases}
$$

where $\mathrm{D}(\epsilon)=\log (1-\epsilon)-\log (\epsilon /(q-1))$.

\section{B. Nonbinary LDPC codes}

We consider next nonbinary LDPC codes defined by an $m \times n$ sparse parity-check matrix $\boldsymbol{H}$ with coefficients in $\mathbb{F}_{q}$. The parity-check matrix can be represented by a Tanner graph with $n$ VNs corresponding to codeword symbols and $m$ CNs corresponding to parity checks. The edge label associated to the edge connecting $\mathrm{v}$ and $\mathrm{c}$ is denoted by $h_{\mathrm{v}, \mathrm{c}}$, with $h_{\mathrm{v}, \mathrm{c}} \in \mathbb{F}_{q} \backslash 0$. The sets $\mathcal{N}(\mathrm{v})$ and $\mathcal{N}(\mathrm{c})$ denote the neighbors of $\mathrm{VN} v$ and $\mathrm{CN}$ c, respectively. Non-binary protograph-based LDPC codes are created from a small Tanner graph called a protograph by a copy-permute-scale procedure [22]. The protograph consists of $n_{0} \mathrm{VNs}$ and $m_{0} \mathrm{CNs}$. Each VN/CN in a protograph defines a variable/check node type. A protograph can be defined by an $m_{0} \times n_{0}$ base matrix $\boldsymbol{B}=\left[b_{i, j}\right]$, where $b_{i, j}$ is the number of edges that connects $\mathrm{v}_{j}$ to $\mathrm{c}_{i}$. In order to obtain the non-binary parity-check matrix $\boldsymbol{H}$, a number of copies of the protograph is generated and their edges are permuted such that connectivity constraints imposed by the base matrix are maintained followed by scaling each edge in the obtained Tanner graph by an element from $\mathbb{F}_{q} \backslash 0$. Note that each VN/CN in a protograph defines a variable/check node type. We consider protograph-based SC-LDPC codes with base matrix in the form [23]

$$
\boldsymbol{B}=\left(\begin{array}{cccc}
\boldsymbol{B}_{0} & & & \\
\boldsymbol{B}_{1} & \boldsymbol{B}_{0} & & \\
\vdots & \boldsymbol{B}_{1} & \boldsymbol{B}_{0} & \\
\boldsymbol{B}_{w} & \vdots & \boldsymbol{B}_{1} & \\
& \boldsymbol{B}_{w} & \vdots & \ddots \\
& & \boldsymbol{B}_{w} & \ddots \\
& & & \ddots
\end{array}\right)
$$

\section{Symbol Message Passing Decoding}

In this section, we describe the proposed SMP algorithm [15] in detail, assuming transmission over the $q$-SC. SMP decoding is an iterative algorithm, where CNs and VNs exchange $q$-ary messages. At the $\ell$ th decoding iteration, let the message sent from $\mathrm{v}$ to $\mathrm{c}$ be $m_{\mathrm{v} \rightarrow \mathrm{c}}^{(\ell)}$, and the message from $\mathrm{c}$ to $\mathrm{v}$ be $m_{\mathrm{c} \rightarrow \mathrm{v}}^{(\ell)}$. Furthermore, the channel observation at $\mathrm{v}$ is denoted by $m_{\mathrm{v}}$. Note that $m_{\mathrm{v} \rightarrow \mathrm{c}}^{(\ell)}, m_{\mathrm{c} \rightarrow \mathrm{v}}^{(\ell)}, m_{\mathrm{v}} \in \mathbb{F}_{q}$. The basic steps of SMP are as follows.

\section{i. Initialization.}

Initially, each $\mathrm{VN} v$ sends to all $\mathrm{c} \in \mathcal{N}(\mathrm{v})$

$$
m_{\mathrm{v} \rightarrow \mathrm{c}}^{(0)}=m_{\mathrm{v}}
$$

where $m_{\mathrm{v}}=y, y$ being the channel observation associated to $\mathrm{VN} \mathrm{v}$.

\section{ii. CN-to-VN step.}

Each CN computes

$$
m_{\mathrm{c} \rightarrow \mathrm{v}}^{(\ell)}=h_{\mathrm{v}, \mathrm{c}}^{-1} \sum_{\mathrm{v}^{\prime} \in \mathcal{N}(\mathrm{c}) \backslash \mathrm{v}} h_{\mathrm{v}^{\prime}, \mathrm{c}} m_{\mathrm{v}^{\prime} \rightarrow \mathrm{c}}^{(\ell-1)}
$$

where the sum and the multiplications are over $\mathbb{F}_{q}$.

iii. VN-to-CN step.

Let $\boldsymbol{E}^{(\ell)}$ be an aggregated extrinsic $\boldsymbol{L}$-vector, with

$$
\begin{aligned}
\boldsymbol{E}^{(\ell)} & =\left[E_{0}^{(\ell)}, E_{1}^{(\ell)}, \ldots, E_{\alpha^{q-2}}^{(\ell)}\right] \\
& =\boldsymbol{L}\left(m_{\mathrm{v}}\right)+\sum_{\mathrm{c}^{\prime} \in \mathcal{N}(\mathrm{v}) \backslash \mathrm{c}} \boldsymbol{L}\left(m_{\mathrm{c}^{\prime} \rightarrow \mathrm{v}}^{(\ell)}\right) .
\end{aligned}
$$

Then, each VN computes

$$
m_{\mathrm{v} \rightarrow \mathrm{c}}^{(\ell)}=\underset{u \in \mathbb{F}_{q}}{\arg \max } E_{u}^{(\ell)} .
$$

Whenever multiple maximizing arguments exist, the arg max function returns one of them at random with uniform probability.

\section{iv. Final decision.}

To estimate its codeword symbol, each VN computes

$$
\hat{x}^{(\ell)}=\underset{u \in \mathbb{F}_{q}}{\arg \max } L_{\mathrm{app}, u}^{(\ell)}
$$

with

$$
\begin{aligned}
\boldsymbol{L}_{\mathrm{app}}^{(\ell)} & =\left[L_{\mathrm{app}, 0}^{(\ell)}, L_{\mathrm{app}, 1}^{(\ell)}, \ldots, L_{\mathrm{app}, \alpha^{q-2}}^{(\ell)}\right] \\
& =\boldsymbol{L}\left(m_{\mathrm{v}}\right)+\sum_{\mathrm{c} \in \mathcal{N}(\mathrm{v})} \boldsymbol{L}\left(m_{\mathrm{c} \rightarrow \mathrm{v}}^{(\ell)}\right) .
\end{aligned}
$$

The VN operation can be interpreted as if the CNs and the channel would vote for the value of the code symbol associated to the VN. The VN assigns different weights to the $\mathrm{CN}$ and channel votes and selects the element with the highest score. 
In (4), the $\boldsymbol{L}$-vector corresponding to the channel observation is obtained from (2) using the channel error probability $\epsilon$. Further, we model each CN-to-VN message, as an observation of the symbol $X$ (associated to v), at the output of an extrinsic $q$-SC channel [3], [24] with crossover probability $\xi^{(\ell)}(\mathrm{c}, \mathrm{v})$, which is the error probability of the message sent over an edge of the type defined by the pair $(c, v)$.

In general, the error probabilities $\xi^{(\ell)}(\mathrm{c}, \mathrm{v})$ are not known. Estimates can be obtained from density evolution (DE) analysis, as proposed in [3], [4], and are then used to compute the corresponding $\boldsymbol{L}$-vectors in (4).

\section{Density Evolution AnAlysis}

Consider a protograph with a $m_{0} \times n_{0}$ base matrix $\boldsymbol{B}$. We derive the DE of the SMP decoder over a $q$-SC with crossover probability $\epsilon$. Due to channel symmetry, we may assume that the all-zeros codeword was transmitted.

Let $p_{a}^{(\ell)}(i, j)$ be the probability that a message sent from a $\mathrm{VN}$ of type $\mathrm{v}_{j}$ to a CN of type $\mathrm{c}_{i}$ on one of the $b_{i, j}$ edges connecting $\mathrm{v}_{j}$ to $\mathrm{c}_{i}$ takes value $a \in \mathbb{F}_{q}$ at the $\ell$-th iteration. Similarly, $s_{a}^{(\ell)}(i, j)$ and denotes the probability that a message sent from $\mathrm{c}_{i}$ to $\mathrm{v}_{j}$ on one of the $b_{i, j}$ edges connecting $\mathrm{c}_{i}$ to $\mathrm{v}_{j}$ takes value $a$. The probability of a correct decision of a VN of type $\mathrm{v}_{j}$ at the $\ell$-th iteration is denoted by $P_{\text {app }}^{(\ell)}(j)$. In the limit of $n \rightarrow \infty$, the DE analysis can be summarized in the following steps.

\section{1) Initialization.}

For $j=1,2, \ldots, n_{0}$ and $i=1,2, \ldots, m_{0}$ with $b_{i, j} \neq 0$, we have

$$
p_{a}^{(0)}(i, j)= \begin{cases}1-\epsilon, & a=0 \\ \frac{\epsilon}{q-1}, & \text { otherwise. }\end{cases}
$$

2) For $\ell=1,2, \ldots, L_{\max }$

\section{Check to variable update}

For $j=1,2, \ldots, n_{0}$ and $i=1,2, \ldots, m_{0}$, if $b_{i, j} \neq 0$ then $s_{0}^{(\ell)}(i, j)$ can be computed from (5), where $\delta_{i j}$ is the Kronecker delta function.

Due to symmetry, we have

$$
s_{a}^{(\ell)}(i, j)=\frac{1-s_{0}^{(\ell)}(i, j)}{q-1}, \quad \forall a \in \mathbb{F}_{q} \backslash 0 .
$$

\section{Variable to check update}

Consider the messages sent from $\mathrm{c}_{i}$ to $\mathrm{v}_{j}$. For each $u \in$ $\mathbb{F}_{q}$, define the random vector

$$
\boldsymbol{F}_{u}^{(\ell)}=\left(F_{u, 1}^{(\ell)}, \ldots, F_{u, m_{0}}^{(\ell)}\right)
$$

and its realization

$$
\boldsymbol{f}_{u}^{(\ell)}=\left(f_{u, 1}^{(\ell)}, \ldots, f_{u, m_{0}}^{(\ell)}\right)
$$

where $F_{u, e}^{(\ell)}$ is the $\mathrm{RV}$ associated to the number of messages equal to $u$ that $\mathrm{c}_{e}$ sends to $\mathrm{v}_{j}$, at the $\ell$-th iteration, on $b_{e, j}-\delta_{e i}$ of the $b_{e, j}$ edges connecting $\mathrm{c}_{e}$ to $\mathrm{v}_{j}$. Thus, for $e=1, \ldots, m_{0}$, we have $\forall u \in \mathbb{F}_{q}$ $0 \leq f_{u, e}^{(\ell)} \leq b_{e, j}-\delta_{e i}$ and $\sum_{u \in \mathbb{F}_{q}} f_{u, e}^{(\ell)}=b_{e, j}-\delta_{e i}$. The elements $E_{u}^{(\ell)}$ of the aggregated extrinsic $\boldsymbol{L}$-vector at the input of $\mathrm{v}_{j}$ are given by

$E_{u}^{(\ell)}= \begin{cases}\mathrm{D}(\epsilon)+\sum_{e: b_{e, j} \neq 0} \mathrm{D}\left(\xi^{(\ell)}(e, j)\right) f_{u, e}^{(\ell)}, & \text { if } u=y \\ \sum_{e: b_{e, j} \neq 0} \mathrm{D}\left(\xi^{(\ell)}(e, j)\right) f_{u, e}^{(\ell)}, & \text { otherwise }\end{cases}$

where

$$
\xi^{(\ell)}(e, j)=1-s_{0}^{(\ell)}(e, j)
$$

We denote by $\mathbb{I}(\mathcal{P})$ the indicator function, which takes value 1 if the proposition $\mathcal{P}$ is true and 0 otherwise. Let $\mathcal{E}^{(\ell)}$ be the set of maximizers of $\boldsymbol{E}^{(\ell)}$, i.e.,

$$
\mathcal{E}^{(\ell)}=\left\{u \in \mathbb{F}_{q} \mid E_{u}^{(\ell)}=\max _{a \in \mathbb{F}_{q}} E_{a}^{(\ell)}\right\} .
$$

Then for $j=1,2, \ldots, n_{0}$ and $i=1,2, \ldots, m_{0}$ with $b_{i, j} \neq 0, p_{0}^{(\ell)}(i, j)$ can be written as shown in (6).

Due to symmetry, for any $a \in \mathbb{F}_{q} \backslash 0$ we have

$$
p_{a}^{(\ell)}(i, j)=\frac{1-p_{0}^{(\ell)}(i, j)}{q-1} .
$$

\section{A posteriori update}

Consider now the probability of a correct decision of a $\mathrm{VN}$ of type $\mathrm{v}_{j}$. We define for each $u \in \mathbb{F}_{q}$ the random vector

$$
\tilde{\boldsymbol{F}}_{u}^{(\ell)}=\left(\tilde{F}_{u, 1}^{(\ell)}, \ldots, \tilde{F}_{u, m_{0}}^{(\ell)}\right)
$$

and its realization

$$
\tilde{\boldsymbol{f}}_{u}^{(\ell)}=\left(\tilde{f}_{u, 1}^{(\ell)}, \ldots, \tilde{f}_{u, m_{0}}^{(\ell)}\right)
$$

where $\tilde{F}_{u, e}^{(\ell)}$ is the $\mathrm{RV}$ associated to the number of messages equal to $u$ that $\mathrm{c}_{e}$ sends to $\mathrm{v}_{j}$, at the $\ell$-th iteration, on the $b_{e, j}$ edges connecting $\mathrm{c}_{e}$ to $\mathrm{v}_{j}$. Thus, for $e=1, \ldots, m_{0}$, we have $\forall u \in \mathbb{F}_{q} 0 \leq \tilde{f}_{u, e}^{(\ell)} \leq b_{e, j}$ and $\sum_{u \in \mathbb{F}_{q}} \tilde{f}_{u, e}^{(\ell)}=b_{e, j}$. The elements $L_{\mathrm{app}, u}^{(\ell)}$ at the input of $\mathrm{v}_{j}$ are given by

$L_{\mathrm{app}, u}^{(\ell)}= \begin{cases}\mathrm{D}(\epsilon)+\sum_{e: b_{e, j} \neq 0} \mathrm{D}\left(\xi^{(\ell)}(e, j)\right) \tilde{f}_{u, e}^{(\ell)}, & \text { if } u=y \\ \sum_{e: b_{e, j} \neq 0} \mathrm{D}\left(\xi^{(\ell)}(e, j)\right) \tilde{f}_{u, e}^{(\ell)}, & \text { otherwise. }\end{cases}$

Let $\mathcal{L}_{\mathrm{app}}^{(\ell)}$ be the set of maximizers of $\boldsymbol{L}_{\mathrm{app}}^{(\ell)}$, i.e.,

$$
\mathcal{L}_{\text {app }}^{(\ell)}=\left\{u \in \mathbb{F}_{q} \mid L_{\mathrm{app}, u}^{(\ell)}=\max _{a \in \mathbb{F}_{q}} L_{\mathrm{app}, a}^{(\ell)}\right\} .
$$

Then for $j=1,2, \ldots, n_{0}, P_{\mathrm{app}}^{(\ell)}(j)$ is obtained from (7).

The protograph ensemble iterative decoding threshold $\epsilon^{\star}$ is defined as the maximum $\epsilon$ for which $P_{\text {app }}^{(\ell)}(j) \rightarrow 1$ for $j=$ $1,2, \ldots, n_{0}$ as $\ell \rightarrow \infty$. 


$$
s_{0}^{(\ell)}(i, j)=\frac{1}{q}\left(1+(q-1) \prod_{e: b_{i, e} \neq 0}\left(\frac{q \cdot p_{0}^{(\ell-1)}(i, e)-1}{q-1}\right)^{b_{i, e}-\delta_{e j}}\right) .
$$

$$
\begin{aligned}
p_{0}^{(\ell)}(i, j)= & \sum_{y \in \mathbb{F}_{q}} \operatorname{Pr}\{Y=y\} \sum_{f_{0}^{(\ell)}, f_{1}^{(\ell)}, \ldots, f_{\alpha q-2}^{(\ell)}}\left[\prod_{e: b_{e, j} \neq 0}\left(\begin{array}{c}
b_{e, j}-\delta_{i e}^{(\ell)} \\
f_{0, e}^{(\ell)}, \ldots, f_{\alpha^{q-2}, e}^{(\ell)}
\end{array}\right)\left(s_{0}^{(\ell)}(e, j)\right)^{f_{0, e}^{(\ell)}} \times\right. \\
& \left.\left(\frac{1-s_{0}^{(\ell)}(e, j)}{q-1}\right)^{b_{e, j}-\delta_{i e}-f_{0, e}^{(\ell)}}\right] \frac{\mathbb{I}\left(0 \in \mathcal{E}^{(\ell)}\right)}{\left|\mathcal{E}^{(\ell)}\right|}
\end{aligned}
$$

$$
\begin{aligned}
P_{\text {app }}^{(\ell)}(j)= & \sum_{y \in \mathbb{F}_{q}} \operatorname{Pr}\{Y=y\} \sum_{\tilde{f}_{0}^{(\ell)}, \tilde{f}_{1}^{(\ell)}, \ldots, \tilde{f}_{\alpha q-2}^{(\ell)}}\left[\prod_{e: b_{e, j} \neq 0}\left(\begin{array}{c}
\tilde{f}_{0, e}^{(\ell)}, \ldots, \tilde{f}_{\alpha^{q-2}, e}^{(\ell)} \\
b_{e, j}
\end{array}\right)\left(s_{0}^{(\ell)}(e, j)\right)^{\tilde{f}_{0, e}^{(\ell)}} \times\right. \\
& \left.\left(\frac{1-s_{0}^{(\ell)}(e, j)}{q-1}\right)^{b_{e, j}-\tilde{f}_{0, e}^{(\ell)}}\right] \frac{\mathbb{I}\left(0 \in \mathcal{L}_{\text {app }}^{(\ell)}\right)}{\left|\mathcal{L}_{\text {app }}^{(\ell)}\right|} .
\end{aligned}
$$

Remark 1. As for the message passing algorithms proposed in [3], [4], DE analysis plays a two-fold role. First, it allows deriving the iterative decoding threshold of the LDPC code ensemble under analysis. Second, it provides (as a byproduct) estimates of the extrinsic channel reliabilities $\xi^{(\ell)}$ to be used in Sec. III. The estimates are accurate when decoding long codes (this is also the regime in which DE analysis captures well the evolution of the message probability distributions).

\section{Thresholds of Selected Ensembles}

We follow the approach of [25] to determine the decoding threshold of protograph-based SC-LDPC code ensembles for window decoding. To this end, we apply the DE analysis of Sec. IV on the protograph matrix $\boldsymbol{B}_{[1: 5 W, 1: W]}$, which is formed from the first $W$ block rows and $W$ block columns of $\boldsymbol{B}$ in (3). We choose $W$ large enough that increasing it further doesn't improve the decoding thresholds. For the following results we fix $W=30$. Convergence of the window decoder is declared when the probability of correct decision for the VNs in the first block column is one. We present the iterative decoding thresholds of protograph based SC-LDPC codes with VN degrees $\mathrm{d}_{\mathrm{v}} \in\{3,4,5,6\}$ for $q \in\{2,4,8,16\}$ and code rates $R \in\{1 / 2,2 / 3,3 / 4,4 / 5\}$. The submatrices $\boldsymbol{B}_{i}$ in (3) of $\boldsymbol{B}_{\mathrm{d}_{\mathrm{v}}, \mathrm{d}_{\mathrm{c}}}^{q}$ are given by

$$
\boldsymbol{B}_{i}=\underbrace{\left(\begin{array}{llll}
1 & 1 & \ldots & 1
\end{array}\right)}_{\mathrm{d}_{\mathrm{c}}}, \quad i=0, \ldots, w
$$

where $w=\mathrm{d}_{\mathrm{v}}-1$. The results are summarized in Tables II, IV, VI and VIII. For the sake of comparison, the iterative decoding thresholds of uncoupled regular LDPC ensembles, which can be computed from the analysis in [15] , are provided in Tables I, III, V and VII.
As expected, a $\left(d_{v}, d_{c}\right)$ SC-LDPC code ensemble attains a remarkably larger threshold than the corresponing uncoupled ensemble. For example, for $q=512$ and $R=1 / 2$, the $(3,6)$ SC-LDPC code ensemble has threshold $\epsilon^{\star}=0.139$, while the $(3,6)$ uncoupled code ensemble has threshold $\epsilon^{\star}=0.11$. Increasing gains can be observed for larger degrees.

\section{Conclusions}

We analyzed the asymptotic performance of symbol message passing (SMP) decoding applied to nonbinary SC-LDPC code ensembles. The analysis, performed via density evolution over the $q$-ary symmetric channel (with $q$ being the field order used for the code construction), shows how regular SC-LDPC code ensembles outperform their uncoupled counterparts also under the simplified message passing decoding algorithm under consideration.

\section{ACKNOWLEDGMENT}

The authors would like to thank Gerhard Kramer for fruitful discussions and comments leading up to this work.

\section{REFERENCES}

[1] R. G. Gallager, "Low-density parity-check codes," Ph.D. dissertation, Dep. Electrical Eng., M.I.T, Cambridge, MA, Jul. 1963.

[2] T. Richardson and R. Urbanke, "The capacity of low-density paritycheck codes under message-passing decoding," IEEE Trans. Inf. Theory, vol. 47, no. 2, pp. 599-618, Feb. 2001.

[3] G. Lechner, T. Pedersen, and G. Kramer, "Analysis and design of binary message passing decoders," IEEE Trans. Commun., vol. 60, no. 3, pp. 601-607, Mar. 2012.

[4] E. B. Yacoub, F. Steiner, B. Matuz, and G. Liva, "Protograph-based LDPC code design for ternary message passing decoding," in Proc. of the 12th International ITG Conf. on Systems, Commun. and Coding, SCC 2019, Rostock, Germany, Feb. 2019.

[5] S. K. Planjery, D. Declercq, L. Danjean, and B. Vasic, "Finite alphabet iterative decoders - part I: Decoding beyond belief propagation on the binary symmetric channel," IEEE Trans. Commun., vol. 61, no. 10, pp. 4033-4045, Oct. 2013. 
[6] L. Barnault and D. Declercq, "Fast decoding algorithm for LDPC over GF $\left(2^{q}\right)$," in Proc. IEEE Inf. Theory Workshop (ITW), Cergy, France, Mar. 2003, pp. 70-73.

[7] D. Declercq and M. Fossorier, "Decoding algorithms for nonbinary LDPC codes over GF (q)," IEEE Trans. Commun., vol. 55, no. 4, pp. 633-643, Apr. 2007.

[8] M. Stark, J. Lewandowsky, S. Saha, and G. Bauch, "Decoding of nonbinary LDPC codes using the information bottleneck method," arXiv preprint arXiv:1810.08921, Oct. 2018.

[9] J. J. Metzner, "Majority-logic-like decoding of vector symbols," IEEE Trans. Commun., vol. 44, no. 10, pp. 1227-1230, Oct. 1996.

[10] M. G. Luby and M. Mitzenmacher, "Verification-based decoding for packet-based low-density parity-check codes," IEEE Trans. Inf. Theory, vol. 51, no. 1, pp. 120-127, Jan. 2005

[11] B. Matuz, G. Liva, E. Paolini, and M. Chiani, "Verification-based decoding with map erasure recovery," in Proc. of the 9th International ITG Conf. on Systems, Commun. and Coding, SCC 2013, München, Germany, Jan. 2013.

[12] A. Shokrollahi and W. Wang, "Low-density parity-check codes with rates very close to the capacity of the q-ary symmetric channel for large q," in Proc. IEEE Int. Symp. Inf. Theory, Chicago, IL, Jun. 2004.

[13] F. Zhang and H. D. Pfister, "Analysis of verification-based decoding on the $q$-ary symmetric channel for large $q$," IEEE Trans. Inf. Theory, vol. 57, no. 10, pp. 6754-6770, 2011.

[14] B. M. Kurkoski, K. Yamaguchi, and K. Kobayashi, "Density evolution for GF $(q)$ LDPC codes via simplified message-passing sets," in Proc. Inf. Theory and Appl. Workshop, Feb. 2007, pp. 237-244.

[15] F. Lázaro, A. Graell i Amat, G. Liva, and B. Matuz, "Symbol Message Passing Decoding of Nonbinary Low-Density Parity-Check Codes," Available on arXiv, 2019.

[16] A. Jimenéz Feltström and K. S. Zigangirov, "Time-varying periodic convolutional codes with low-density parity-check matrix," IEEE Trans. Inf. Theory, vol. 45, no. 6, pp. 2181-2191, Sep. 1999.

[17] M. Lentmaier, A. Sridharan, D. J. Costello, and K. S. Zigangirov, "Iterative decoding threshold analysis for LDPC convolutional codes," IEEE Trans. Inf. Theory, vol. 56, no. 10, pp. 5274-5289, Oct. 2010.

[18] S. Kudekar, T. J. Richardson, and R. L. Urbanke, "Threshold saturation via spatial coupling: Why convolutional LDPC ensembles perform so well over the BEC," IEEE Trans. Inf. Theory, vol. 57, no. 2, pp. 803834, Feb. 2011

[19] S. Kudekar, T. Richardson, and R. L. Urbanke, "Spatially coupled ensembles universally achieve capacity under belief propagation," IEEE Trans. Inf. Theory, vol. 59, no. 12, pp. 7761-7813, Dec. 2013.

[20] I. Andriyanova and A. Graell i Amat, "Threshold saturation for nonbinary SC-LDPC codes on the binary erasure channel," IEEE Trans. Inf. Theory, vol. 62, no. 5, pp. 2622-2638, May 2016.

[21] F. Steiner, E. B. Yacoub, B. Matuz, G. Liva, and A. Graell i Amat, "One and two bit message passing for SC-LDPC codes with higher-order modulation," submitted to IEEE/OSA J. Lightw. Technol., 2019. [Online]. Available: http://arxiv.org/abs/1902.10391

[22] L. Dolecek, D. Divsalar, Y. Sun, and B. Amiri, "Non-Binary ProtographBased LDPC Codes: Enumerators, Analysis, and Designs," IEEE Trans. Inf. Theory, vol. 60, no. 7, Jul. 2014.

[23] D. G. M. Mitchell, M. Lentmaier, and D. J. Costello, "Spatially Coupled LDPC Codes Constructed From Protographs," IEEE Trans. Inf. Theory, vol. 61 , no. 9 , pp. $4866-4889$, Sep. 2015.

[24] A. Ashikhmin, G. Kramer, and S. ten Brink, "Extrinsic information transfer functions: model and erasure channel properties," IEEE Trans. Inf. Theory, vol. 50, no. 11, pp. 2657-2673, Nov. 2004.

[25] A. Iyengar, M. Papaleo, P. Siegel, J. Wolf, A. Vanelli-Coralli, and G. Corazza, "Windowed Decoding of Protograph-Based LDPC Convolutional Codes Over Erasure Channels," IEEE Trans. Inf. Theory, vol. 58, no. 4, pp. 2303-2320, Apr. 2012.
TABLE I

THRESHOLDS FOR VARIOUS RATE- $1 / 2$ REGULAR ENSEMBLES AND DIFFERENT $q$.

\begin{tabular}{rccccl}
\hline$q$ & $(3,6)$ & $(4,8)$ & $(5,10)$ & $(6,12)$ & $\epsilon_{\text {Sh }}$ \\
\hline 2 & 0.0395 & 0.0516 & 0.0416 & 0.0396 & 0.11 \\
4 & 0.0890 & 0.0814 & 0.0811 & 0.0738 & 0.1893 \\
8 & 0.1039 & 0.1064 & 0.1006 & 0.1010 & 0.2470 \\
16 & 0.1075 & 0.1371 & 0.1163 & 0.1122 & 0.2897 \\
32 & 0.1092 & 0.1637 & 0.1361 & 0.1209 & 0.3217 \\
64 & 0.1101 & 0.1759 & 0.1613 & 0.1351 & 0.3462 \\
128 & 0.1105 & 0.1818 & 0.1770 & 0.1555 & 0.3653 \\
256 & 0.1107 & 0.1846 & 0.1846 & 0.1703 & 0.3805 \\
512 & 0.1108 & 0.1861 & 0.1883 & 0.1775 & 0.3927 \\
\hline
\end{tabular}

TABLE II

THRESHOLDS FOR VARIOUS RATE- $1 / 2$ SPATIALLY COUPLED ENSEMBLES AND DIFFERENT $q$.

\begin{tabular}{rccccl}
\hline$q$ & $\boldsymbol{B}_{3,6}^{q}$ & $\boldsymbol{B}_{4,8}^{q}$ & $\boldsymbol{B}_{5,10}^{q}$ & $\boldsymbol{B}_{6,12}^{q}$ & $\epsilon_{\mathrm{Sh}}$ \\
\hline 2 & 0.0542 & 0.0626 & 0.0680 & 0.0702 & 0.11 \\
4 & 0.0942 & 0.1111 & 0.1192 & 0.1226 & 0.1893 \\
8 & 0.1183 & 0.1475 & 0.1571 & 0.1612 & 0.2470 \\
16 & 0.1288 & 0.1742 & 0.1851 & 0.1897 & 0.2897 \\
32 & 0.1340 & 0.1931 & 0.2056 & 0.2104 & 0.3217 \\
64 & 0.1366 & 0.2062 & 0.2201 & 0.2255 & 0.3462 \\
128 & 0.1380 & 0.2135 & 0.2298 & 0.2360 & 0.3653 \\
256 & 0.1386 & 0.2171 & 0.2363 & 0.2430 & 0.3805 \\
512 & 0.1390 & 0.2190 & 0.2404 & 0.2476 & 0.3927 \\
\hline
\end{tabular}

TABLE III

THRESHOLDS FOR VARIOUS RATE- $2 / 3$ REGULAR ENSEMBLES AND DIFFERENT $q$.

\begin{tabular}{rccccl}
\hline$q$ & $(3,9)$ & $(4,12)$ & $(5,15)$ & $(6,18)$ & $\epsilon_{\mathrm{Sh}}$ \\
\hline 2 & 0.0155 & 0.0279 & 0.0246 & 0.0230 & 0.0615 \\
4 & 0.0382 & 0.0457 & 0.0489 & 0.0438 & 0.1084 \\
8 & 0.0621 & 0.0584 & 0.0621 & 0.0606 & 0.1442 \\
16 & 0.0645 & 0.0746 & 0.0700 & 0.0723 & 0.1718 \\
32 & 0.0655 & 0.0955 & 0.0799 & 0.0760 & 0.1934 \\
64 & 0.0661 & 0.1094 & 0.0942 & 0.0823 & 0.2104 \\
128 & 0.0663 & 0.1157 & 0.1102 & 0.0933 & 0.2241 \\
256 & 0.0664 & 0.1187 & 0.1185 & 0.1071 & 0.2351 \\
512 & 0.0665 & 0.1202 & 0.1225 & 0.1149 & 0.2443 \\
\hline
\end{tabular}

TABLE IV

THRESHOLDS FOR VARIOUS RATE- $2 / 3$ SPATIALLY COUPLED ENSEMBLES AND DIFFERENT $q$.

\begin{tabular}{rccccl}
\hline$q$ & $\boldsymbol{B}_{3,9}^{q}$ & $\boldsymbol{B}_{4,12}^{q}$ & $\boldsymbol{B}_{5,15}^{q}$ & $\boldsymbol{B}_{6,18}^{q}$ & $\epsilon_{\mathrm{Sh}}$ \\
\hline 2 & 0.0239 & 0.0333 & 0.0373 & 0.0393 & 0.0615 \\
4 & 0.0461 & 0.0607 & 0.0673 & 0.0703 & 0.1084 \\
8 & 0.0646 & 0.0825 & 0.0907 & 0.0944 & 0.1442 \\
16 & 0.0720 & 0.0999 & 0.1089 & 0.1129 & 0.1718 \\
32 & 0.0752 & 0.1135 & 0.1231 & 0.1272 & 0.1934 \\
64 & 0.0768 & 0.1239 & 0.1338 & 0.1381 & 0.2104 \\
128 & 0.0775 & 0.1306 & 0.1416 & 0.1463 & 0.2241 \\
256 & 0.0779 & 0.1340 & 0.1471 & 0.1521 & 0.2351 \\
512 & 0.0781 & 0.1356 & 0.1509 & 0.1561 & 0.2443 \\
\hline
\end{tabular}


TABLE V

THRESHOLDS FOR VARIOUS RATE-3/4 REGULAR ENSEMBLES AND DIFFERENT $q$.

\begin{tabular}{rlllll}
\hline$q$ & $(3,12)$ & $(4,16)$ & $(5,20)$ & $(6,24)$ & $\epsilon_{\text {Sh }}$ \\
\hline 2 & 0.0082 & 0.0175 & 0.0173 & 0.0159 & 0.0417 \\
4 & 0.0212 & 0.0311 & 0.0330 & 0.0306 & 0.0744 \\
8 & 0.0396 & 0.0390 & 0.0450 & 0.0420 & 0.1001 \\
16 & 0.0460 & 0.0492 & 0.0499 & 0.0528 & 0.1203 \\
32 & 0.0468 & 0.0630 & 0.0557 & 0.0558 & 0.1363 \\
64 & 0.0472 & 0.0772 & 0.0647 & 0.0589 & 0.1492 \\
128 & 0.0474 & 0.0839 & 0.0772 & 0.0654 & 0.1597 \\
256 & 0.0475 & 0.0870 & 0.0860 & 0.0755 & 0.1684 \\
512 & 0.0475 & 0.0885 & 0.0901 & 0.0837 & 0.1756 \\
\hline
\end{tabular}

TABLE VI

THRESHOLDS FOR VARIOUS RATE-3/4 SPATIALLY COUPLED ENSEMBLES AND DIFFERENT $q$

\begin{tabular}{rccccl}
\hline$q$ & $\boldsymbol{B}_{3,12}^{q}$ & $\boldsymbol{B}_{4,16}^{q}$ & $\boldsymbol{B}_{5,20}^{q}$ & $\boldsymbol{B}_{6,24}^{q}$ & $\epsilon_{\mathrm{Sh}}$ \\
\hline 2 & 0.0134 & 0.0218 & 0.0249 & 0.0266 & 0.0417 \\
4 & 0.0278 & 0.0404 & 0.0457 & 0.0483 & 0.0744 \\
8 & 0.0415 & 0.0556 & 0.0624 & 0.0656 & 0.1001 \\
16 & 0.0494 & 0.0683 & 0.0758 & 0.0792 & 0.1203 \\
32 & 0.0519 & 0.0790 & 0.0866 & 0.0901 & 0.1363 \\
64 & 0.0531 & 0.0875 & 0.0952 & 0.0987 & 0.1492 \\
128 & 0.0536 & 0.0938 & 0.1018 & 0.1055 & 0.1597 \\
256 & 0.0539 & 0.0970 & 0.1066 & 0.1105 & 0.1684 \\
512 & 0.0541 & 0.0987 & 0.1100 & 0.1141 & 0.1756 \\
\hline
\end{tabular}

TABLE VII

THRESHOLDS FOR VARIOUS RATE-4/5 REGULAR ENSEMBLES AND DIFFERENT $q$.

\begin{tabular}{rccccl}
\hline$q$ & $(3,15)$ & $(4,20)$ & $(5,25)$ & $(6,30)$ & $\epsilon_{\text {Sh }}$ \\
\hline 2 & 0.005 & 0.0122 & 0.0132 & 0.0120 & 0.0311 \\
4 & 0.0135 & 0.0234 & 0.0245 & 0.0234 & 0.0560 \\
8 & 0.0260 & 0.0289 & 0.0351 & 0.0318 & 0.0758 \\
16 & 0.0358 & 0.0360 & 0.0388 & 0.0412 & 0.0917 \\
32 & 0.0364 & 0.0457 & 0.0425 & 0.0443 & 0.1044 \\
64 & 0.0367 & 0.0581 & 0.0486 & 0.0459 & 0.1148 \\
128 & 0.0368 & 0.0651 & 0.0577 & 0.0500 & 0.1233 \\
256 & 0.0369 & 0.0684 & 0.0666 & 0.0572 & 0.1303 \\
512 & 0.0369 & 0.0699 & 0.0709 & 0.0651 & 0.1362 \\
\hline
\end{tabular}

TABLE VIII

THRESHOLDS FOR VARIOUS RATE- $4 / 5$ SPATIALLY COUPLED ENSEMBLES AND DIFFERENT $q$.

\begin{tabular}{rccccl}
\hline$q$ & $\boldsymbol{B}_{3,15}^{q}$ & $\boldsymbol{B}_{4,20}^{q}$ & $\boldsymbol{B}_{5,25}^{q}$ & $\boldsymbol{B}_{6,30}^{q}$ & $\epsilon_{\mathrm{Sh}}$ \\
\hline 2 & 0.0086 & 0.0158 & 0.0184 & 0.0198 & 0.0311 \\
4 & 0.0187 & 0.0296 & 0.0341 & 0.0363 & 0.0560 \\
8 & 0.0291 & 0.0411 & 0.0469 & 0.0497 & 0.0758 \\
16 & 0.0372 & 0.0510 & 0.0575 & 0.0604 & 0.0917 \\
32 & 0.0394 & 0.0598 & 0.0662 & 0.0692 & 0.1044 \\
64 & 0.0404 & 0.0670 & 0.0733 & 0.0763 & 0.1148 \\
128 & 0.0409 & 0.0726 & 0.0790 & 0.0821 & 0.1233 \\
256 & 0.0411 & 0.0759 & 0.0833 & 0.0865 & 0.1303 \\
512 & 0.0412 & 0.0775 & 0.0864 & 0.0897 & 0.1362 \\
\hline
\end{tabular}

